\title{
A theory for the estimation of surface fluxes in simple katabatic flows
}

\author{
By BRANKO GRISOGONO ${ }^{1 *}$ and JOHANNES OERLEMANS ${ }^{2}$ \\ ${ }^{1}$ Stockholm University, Sweden \\ ${ }^{2}$ Utrecht University, the Netherlands
}

(Received 19 July 2000; revised 15 May 2001)

\section{SUMMARY}

The classical Prandtl model describes the basic dynamics of katabatic flows, but it fails in determining the gradients near the surface because the eddy diffusivity, $K$, is independent of height, $z$. An improvement is proposed to the Prandtl model by using the WKB method to solve the dynamic equation for any prescribed, reasonably smooth, $K(z)$ profile.

The surface fluxes are obtained by extrapolation from the katabatic jet down to the surface, thus the fluxes depend primarily on the jet which is well captured by the WKB method. The profiles of wind, temperature, momentum and heat fluxes, as well as the surface fluxes calculated using the model, are tested against observations from a meteorological field campaign on the Pasterze glacier, Austria, in 1994, and their numerical simulations. Generally there is a good agreement between observations and theory.

KEYWORDS: Low-level jet WKB method

\section{INTRODUCTION}

The properties of the atmospheric boundary layer over glacier surfaces have recently received increasing attention. This is inspired by the notion that in a warming world glaciers could make a significant contribution to sea-level change. Although meteorological experiments on glaciers show that the largest contribution to the melt energy comes from solar radiation (e.g. Oerlemans et al. 1999), the sensitivity of the melt rate to the temperature change is determined by changes in the long-wave balance and in the turbulent heat flux. Changes in the large-scale state of the atmosphere affect the glacier surface, but only after being modified by usually shallow boundary layers. Observations over glaciers show that katabatic flows are very persistent (e.g. Oerlemans and Vugts 1993; Greuell et al. 1997; Oerlemans et al. 1999). Even for glaciers that are much exposed to large-scale systems, like Vatnajökull on Iceland, the near-surface wind field is almost always generated by katabatic forcing. The persistence of the katabatic flow is illustrated in Fig. 1, showing data from Morteratschgletscher in Switzerland (Oerlemans 2000). The flow is almost always down the local glacier slope. Only during extreme events is the flow over the glacier influenced by the synoptic-scale wind; otherwise, the correlation between the station data and the large-scale wind is very small. Katabatic flow over melting glaciers is characterized by extreme stratification. It is not unusual to measure temperature differences of $15 \mathrm{~K}$ between the glacier surface and a sensor at $3 \mathrm{~m}$ height. The related wind speed is of the order of $5 \mathrm{~m} \mathrm{~s}^{-1}$ and induced by the strong katabatic forcing. The turbulence that creates the heat flux to the surface is generated by the katabatic flow and vice versa. The katabatic flow acts as a heat pump for the glacier. In summary, to quantify the sensitivity of glacier mass balance to temperature change, we need to know how the turbulent exchange is related to the conditions in the ambient atmosphere. Clearly, this cannot be achieved without a thorough understanding of the katabatic flow structure.

Katabatic flows largely determine the surface fluxes of momentum, heat and moisture. Various data analyses have shown that standard profile methods are not suitable for determining the fluxes in katabatic regimes (Munro 1989; Van den Broeke 1997;

* Corresponding author: MISU, Arrhenius Laboratory, S-10691 Stockholm, Sweden. e-mail: branko@misu.su.se

(C) Royal Meteorological Society, 2001. 

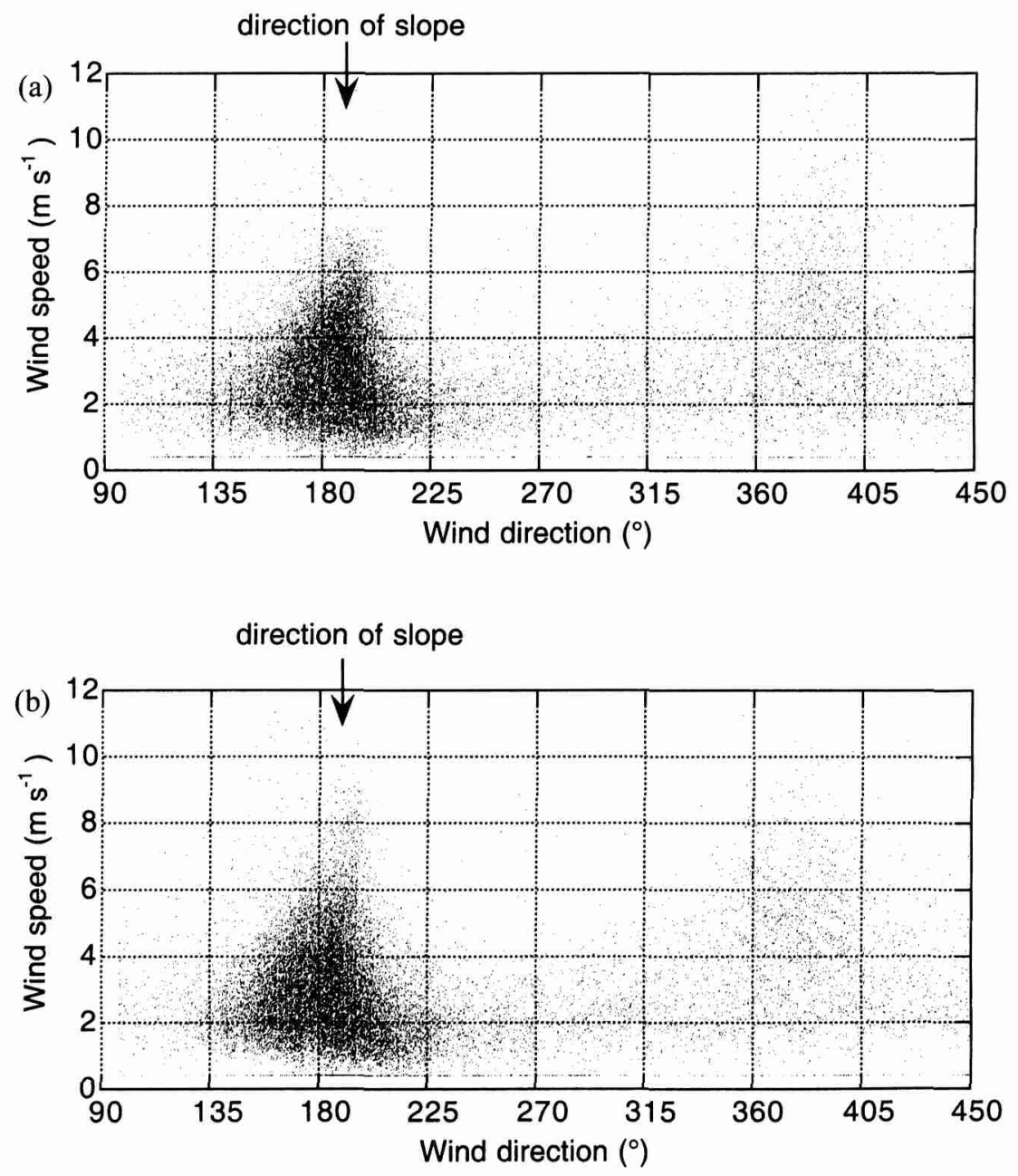

Figure 1. Wind data from an automated weather station located on the snout of the Morteratschgletscher, Switzerland between (a) 1 October 1996 and 30 September 1997 and (b) 1 October 1997 and 30 September 1998. The sensor height is about $3.5 \mathrm{~m}$ (less in winter due to snow cover). Shown are 30-minute mean values of wind direction versus speed. Each graph contains about 17520 data points thus adding to the motivation for this study.

Oerlemans 1998). In the profile analysis, very strong stratification of the flow leads to such large stability corrections that a zero reference problem occurs: the surface fluxes become very sensitive to how the displacement height is actually chosen (Smeets $\mathrm{et} \mathrm{al}$. 1999). We believe that the problem can only be solved by retaining the coupling between thermodynamics and dynamics, which is not done in the standard profile analysis. The simplest model in which this coupling is retained is the Prandtl model for katabatic flow (Prandtl 1942). It consists of two coupled linear diffusion equations, one for momentum and one for temperature, that produce temperature and wind profiles that are in qualitative agreement with observations. However, the Prandtl solution can be criticized for the oversimplified treatment of the turbulent diffusion. Because the eddy diffusivity in the classic Prandtl model does not depend on height, the gradients near the surface are much too small. Katabatic flows belong to 'problematic flows', according to Mahrt (1998), as this type of the very stable boundary layer (SBL) is still principally unsolved. 
This paper intends to add to our knowledge about the katabatic type of the SBL where the basic theory of Monin and Obukhov usually fails (e.g. Zilitinkevich and Calanca 2000).

Numerical modelling of katabatic flows also has its problems. The simpler turbulence schemes do not provide sufficient turbulent diffusion around and through the low-level jet, thus a higher-order scheme becomes a modelling necessity (Arritt and Pielke 1986; Nappo and Rao 1987; Denby 1999). To our knowledge, a successful largeeddy simulation of katabatic flows, in which the shape of observed jets is reproduced accurately, has not yet been carried out.

In view of this, there is room for analytical modelling, in which the constraint of constant eddy diffusivity is relaxed. We have described previously an analytic solution, obtained with the $\mathrm{WKB}^{*}$ method, in which the eddy diffusivity is a smooth prescribed function of height (Grisogono and Oerlemans 2001; hereafter GO). In this case the agreement with observations is significantly better as compared with the linear Prandtl solution.

In this paper we develop the theory further and pay attention to the estimation of the surface fluxes in katabatic flows. We investigate how the friction velocity, $u_{*}$, and temperature scale, $\Theta_{*}$, depend on the characteristics of the katabatic flow, e.g. the lowlevel jet positioned at height $z_{\mathrm{J}}$. Needless to say, the classic surface-layer theory of Monin and Obukhov fails for shallow katabatic flows where the wind maximum occurs at a few metres above the surface, e.g. $z_{\mathrm{J}} \sim 5 \mathrm{~m}$, and other very stable conditions (e.g. Mahrt 1998; Zilitinkevich and Calanca 2000; Pahlow et al. 2001).

The problem reads as follows. After finding the potential temperature, $\Theta$, at height $z$, and wind perturbations for the given terrain slope $\alpha$, the background potentialtemperature lapse rate $\gamma$ and guessed eddy diffusivity $K(z)$, assign the roughness length, $z_{0}$, and estimate the main surface flux parameters:

$$
\left(\alpha, \gamma, K(z) ; z_{0}\right) \rightarrow(\Theta(z), u(z)) \rightarrow\left(\Theta_{*}, u_{*}\right),
$$

where $u$ is the katabatic wind. Consequently, this is an extension and application of the method and results from $\mathrm{GO}$ where $\Theta(z)$ and $u(z)$ are readily obtained. The structure of this paper is as follows: after the model description and a data comparison (taken from $\mathrm{GO})$, a procedure for $\left(\Theta_{*}, u_{*}\right)$ estimation is described, simple approximate expressions are derived, and an example from the data is provided. The study can be useful for boundary-layer analyses, glaciology, hydrology and climatology, ranging from data interpretation to parametrizations of the SBL.

\section{THE KATABATIC MODEL}

\section{(a) The constant-K model of Prandtl}

A balance between the buoyancy force and friction in a simple katabatic flow implies the model of Prandtl (1942) (see, for example, Defant (1949); Mahrt (1982) and Egger (1990)). A steady, one-dimensional Boussinesq, hydrostatic, irrotational flow is assumed and the $K$-theory is employed. The momentum and thermodynamic equations for the perturbations of $\Theta$ and $u$ are:

$$
\begin{gathered}
-g\left(\frac{\Theta}{\Theta_{0}}\right) \sin (\alpha)=\frac{\mathrm{d}\left(K_{\mathrm{M}} \mathrm{d} u / \mathrm{d} z\right)}{\mathrm{d} z} \\
u \gamma \sin (\alpha)=\frac{\mathrm{d}\left(K_{\mathrm{H}} \mathrm{d} \Theta / \mathrm{d} z\right)}{\mathrm{d} z},
\end{gathered}
$$


where $g$ is gravity, $K_{\mathrm{M}}$ and $K_{\mathrm{H}}$ are eddy viscosity and conductivity respectively and $\Theta_{0}$ a reference constant potential temperature. The boundary conditions are: $\Theta(0)=C<0$, where $C$ is surface temperature deficit, $\Theta(z \rightarrow \infty)=0, u(0)=0, u(z \rightarrow \infty)=0$. The $z$-axis is perpendicular to the surface ( $x$-axis) sloped with a small angle $\alpha$ from the horizontal. Note that $\Theta$ is potential temperature deficit (i.e. the actual minus the background potential temperature, $\theta$, the latter also defines the constant potentialtemperature lapse rate $\gamma$ ) and $u$ flows downslope.

For $K_{\mathrm{M}}$ and $K_{\mathrm{H}}$ flow-independent, the system (2.1) is linear. If the turbulent Prandtl number, $P r$, is constant, $P r \equiv K_{\mathrm{M}} / K_{\mathrm{H}}=$ constant, one arrives at a single, linear, fourthorder differential equation. Equivalently, for either $\Theta$ or $u$ (here written for $\Theta$ ) and $K \equiv K_{\mathrm{H}}$, the governing equation becomes:

$$
\Theta^{(4)}+\frac{4 K^{(1)}}{K} \cdot \Theta^{(3)}+f_{2} \cdot \Theta^{(2)}+f_{1} \cdot \Theta^{(1)}+\left(\frac{\sigma_{0}}{K}\right)^{2} \cdot \Theta=0
$$

with

$$
\begin{gathered}
\sigma_{0}^{2} \equiv \frac{g \gamma \sin ^{2}(\alpha)}{\left(\Theta_{0} \operatorname{Pr}\right)} \equiv \frac{(N \sin (\alpha))^{2}}{\operatorname{Pr}} \equiv \frac{N_{\alpha}^{2}}{\operatorname{Pr}} \\
f_{1} \equiv \frac{K^{(3)}}{K}+\frac{K^{(1)} K^{(2)}}{K^{2}}, \quad f_{2} \equiv \frac{3 K^{(2)}}{K}+2\left(\frac{K^{(1)}}{K}\right)^{2},
\end{gathered}
$$

where the superscript brackets represent the derivatives in the $z$ direction, i.e. ()$^{(n)} \equiv$ $\mathrm{d}^{n}() / \mathrm{d} z^{n}, n=1, \ldots, 4, N$ is the background buoyancy frequency and $N_{\alpha}$ its 'slope projection'. The second, third and fourth terms in (2.2a) determine 'secondary $K$-effects' on the katabatic flow and are initially neglected. In other words, the classic constant- $K$ solution is obtained by setting $K^{(n)}=0, n=1,2,3$, in (2.2). Then $f_{1}$ and $f_{2}$ are zero, and only the first and last term in (2.2a) with its constant coefficient remain, yielding the solution to this zero-order-approximated problem (e.g. Egger 1990; Oerlemans 1998):

$$
\begin{gathered}
\Theta_{K=\mathrm{const}}=C \exp \left(-\sigma_{\mathrm{C}} z\right) \cos \left(\sigma_{\mathrm{C}} z\right) \\
u_{K=\mathrm{const}}=-C \mu \exp \left(-\sigma_{\mathrm{C}} z\right) \sin \left(\sigma_{\mathrm{C}} z\right)
\end{gathered}
$$

where $\sigma_{\mathrm{C}}$ is the vertical wave number determining the classical length-scale, and $\mu$ is yet another flow parameter, i.e.

$$
\begin{aligned}
\sigma_{\mathrm{C}}^{2} & \equiv \frac{N_{\alpha}}{2 \operatorname{Pr}^{1 / 2} K}=\frac{\sigma_{0}}{2 K} \\
\mu & \equiv\left(\frac{g}{\Theta_{0} \operatorname{Pr} \gamma}\right)^{1 / 2} .
\end{aligned}
$$

The solution (2.3) is mathematically equivalent to that for the Ekman layer. A complex, dimensionless, katabatic flow function containing $(2.3 \mathrm{a}, \mathrm{b})$ is written as

$$
F_{\mathrm{C}} \equiv\left(\Theta_{K=\mathrm{const}}, u_{K=\mathrm{const}}\right)_{\mathrm{D}-\mathrm{LESS}}=\exp \left\{-(1+\mathrm{i}) \sigma_{\mathrm{C}} z\right\},
$$

where $i$ is $\sqrt{-1}$.

This $F_{\mathrm{C}}$ contains most of the necessary information about the structure of the classic katabatic flow. However, as already mentioned, the surface fluxes obtained from $F_{\mathrm{C}}$ generally do not represent those based on observations (Munro 1989; Oerlemans 1998). Hence, the GO model, an improvement of the Prandtl model, will be introduced and used instead. 


\section{(b) The GO model and the WKB assumption}

The GO model employs the WKB technique (e.g. Bender and Orszag 1978; Gill 1982; Grisogono 1995) to approximate the solution to the governing equation with gradually varying coefficients. The basic assumption is that the scale of the background variability, that is the scale of $K(z)$ here, must be larger than that for the calculated perturbed quantity, $F \equiv(\Theta, u)$. In Grisogono (1995) that is explained for the variability in the Ekman layer, while in GO this WKB scale restriction is related to the katabatic SBL. Numerical simulations show that the elevated maximum of the turbulent kinetic energy (TKE) is above the pure katabatic wind maximum, the latter corresponding to the TKE minimum (Nappo and Rao 1987; Stull 1988, Fig. 12.23, p. 535; Denby 1999, Fig. 3, p. 83). If $K \sim \lambda$ (TKE) ${ }^{1 / 2}$ ( $\lambda$ is the characteristic length-scale) where TKE generally decays with height after its maximum, then $\max (K)$ is usually reached (at least slightly) above the level $z_{\mathrm{TKE}}=\max (\mathrm{TKE})$; hence, $\max (K)$ usually occurs above the katabatic wind maximum. The latter statement complies with the WKB requirement and this is why the WKB method would work for pure katabatic flows. The SBL is split into the inner region with vertically increasing $K(z)$ from $K(z=0)=0$ to its maximum $K_{h}$ at the level $h$, and the outer region with vertically decaying $K(z)$ from $K_{h}$. This is re-addressed in subsection 3(a).

The simplified governing equation now for a first-order-approximated problem is:

$$
F^{(4)}+\frac{4 K^{(1)}}{K} \cdot F^{(3)}+\left(\frac{\sigma_{0}}{K}\right)^{2} \cdot F=0 .
$$

This is solved in GO upward from the height $h, h \equiv h\left(K_{h}\right)=h[\max (K)]$ with the firstorder, and below $h$ with the zero-order WKB method (i.e. dropping the second term in (2.4) but letting $K$ vary in the last term), respectively. A simple patching of these two regional solutions gives a global, asymptotically smooth, uniform solution - the main ingredient for this study:

$$
F \sim\left\{\begin{array}{ll}
\exp \left(-(1+\mathrm{i})\left(\frac{\sigma_{0}}{2}\right)^{1 / 2} \int_{0}^{z} K^{-1 / 2} \mathrm{~d} z\right), & z \leq h \\
\left(\frac{K_{h}}{K(z)}\right)^{1 / 4} \exp \left(-(1+\mathrm{i})\left(\frac{\sigma_{0}}{2}\right)^{1 / 2} \int_{0}^{z} K^{-1 / 2} \mathrm{~d} z\right), & z \geq h
\end{array}\right\} .
$$

The main properties of (2.5) are:

- Generalization of, and similarity with, the constant- $K$ solution, (2.3),

- A more universal form of the vertical length-scale, $H$, where $u$ goes to zero, given implicitly now as:

$$
\pi=\left(\frac{\sigma_{0}}{2}\right)^{1 / 2} \int_{0}^{H} K^{-1 / 2} \mathrm{~d} z
$$

- Sharp contrast with the constant- $K$ solution near the surface; there $F$ now shows a rapid growth for $0<z \ll h$.

For small $z$ (2.5) changes rapidly compared with the equivalent expansion of (2.3e) that exhibits only the linear growth with small $z$. It is exactly this property of $F$, i.e. the sharp near-surface gradients, that will be used to estimate $\left(\Theta_{*}, u_{*}\right)$. The weakness of this method is in the prescription of $K$ instead of being a function of the flow variables, namely $K(\theta, u)$. This problem can be alleviated in a nonlinear model. 


\section{THE $K(z)$ PROFILE AND DATA COMPARISON}

Here we address in (a) a choice of $K(z)$ complying with the WKB scale requirement, a test against numerical solution for a pure katabatic flow, and in (b) a comparison against observations.

\section{(a) $K(z)$ profile and katabatic solutions}

A physically reasonable $K(z)$ must satisfy: $K(z \rightarrow 0) \rightarrow 0$ and again $K(z \gg H) \rightarrow$ 0 while reaching its maximum $K_{h}$ around $H / 4 \leq z \leq 3 H / 4$. It is convenient to choose an O'Brien (1970) $K(z)$ type of profile, see, for example, Pielke (1984) and Stull (1988, pp. 209-210). The $K(z)$ profile due to O'Brien (1970) is generalized and simplified here and in GO into a linear-exponential function which, if expanded for small $z$, approximates the O'Brien third-order polynomial in $z$. The assumed $K(z)$ profile is:

$$
K(z)=\text { constant } z \exp \left\{-0.5(z / h)^{2}\right\} .
$$

This $K(z)$ will be used in (2.5); 'constant' can be equal to, for example, $\mathrm{e}^{1 / 2} \max [K(z)]$ $/ h$. Needless to say, $K(z)$ is not limited to $(3.1)$ and other $K(z)$ are clearly possible provided they satisfy the WKB validity criterion, i.e. $h>\max [D(\Theta), D(u)]=$ $D(F), D$ being the respective scale-height. Here $h$ scales roughly with the inverse of (2.3c) as a first guess. Essentially, $K$ must reach its maximum somewhere in the SBL; also $h \geq z_{\mathrm{TKE}}>z_{\mathrm{J}}(\sim D(u))$ implies from Nappo and Rao (1987), Stull (1988), Denby (1999), and others. The same studies also suggest that $D(\Theta) \sim D(u)$ in pure katabatic flows*. Returning back to the justification of (3.1), we recall that for katabatic flows the $\min (\mathrm{TKE})$ occurs at $z_{\mathrm{J}}$. Furthermore, $z_{\mathrm{TKE}}>z_{\mathrm{J}}$, while the lower TKE maximum occurs between the jet and the surface. Higher-order closure numerical models often parametrize $K \sim \lambda$ (TKE) $)^{1 / 2}$ with $\lambda$ usually either of a Blackadar type, $\left(\lambda_{\mathrm{B}}=k z \lambda_{\infty} /\left(k z+\lambda_{\infty}\right)\right.$, where $k$ is the von Kármán constant and $\lambda_{\infty}$ an asymptotic length) or of a ' $z$-less stratification' type $\left(\lambda_{\mathrm{N}} \sim(\mathrm{TKE})^{1 / 2} / N_{\text {LOC }}\right.$, where $N_{\text {LOC }}$ is the local buoyancy frequency). For smoothness, many models choose the $\min \left(\lambda_{B}, \lambda_{N}\right)$. Thus, either TKE $\sim\left(K / \lambda_{\mathrm{B}}\right)^{2}$, or TKE $\sim K N_{\mathrm{LOC}}$. It is possible to show for TKE $=\mathrm{TKE}_{\mathrm{B}} \sim$ $\left(K / \lambda_{\mathrm{B}}\right)^{2}$ that $\max \left(\mathrm{TKE}_{\mathrm{B}}\right)$ is below $\max (K)$, i.e. $z_{\mathrm{TKE}} \leq h$. In the latter case, where $\mathrm{TKE}=\mathrm{TKE}_{\mathrm{N}} \sim K N_{\mathrm{LOC}}$, it is more difficult to show explicitly that $h \geq z_{\mathrm{TKE}}$; however, this becomes less cumbersome if $\mathrm{d} \Theta / \mathrm{d} z$ entering $N_{\mathrm{LOC}}$ is simply taken from the classic Prandtl solution. Anyhow, it appears usually that max(TKE) is at least somewhat below $\max (K)$. Thus, $h \geq z_{\max (\mathrm{TKE})}>z_{\mathrm{J}}$, as required. Finally, note that the $K$ profiles modelled in this way qualitatively agree with our (3.1) and that $h>z_{\mathrm{J}}$. Hence, the WKB scale requirement for (3.1) and the katabatic flow, namely $h>D$ or at least $h \geq D$, is justified, and it is claimed that (3.1) is a simple yet physically based $K$ formulation for pure katabatic flows.

An example in Fig. 2 compares the constant- $K$ and WKB against a numerical solution for a katabatic flow. The numerical solution to the basic problem (2.1) using (3.1) is obtained using the Adams-Bashforth scheme in time $t$ (e.g. Durran 1999) and centred in space until a steady state is reached; the scheme's accuracy is $\mathrm{O}\left[(\Delta t)^{3},(\Delta z)^{2}\right]$ yielding the most accurate solution here. The constant- $K$ solution is different from the WKB solution. While the WKB solution closely resembles the numerical solution, that cannot be said for the constant- $K$ solution. A few other $K(z)$ profiles are tried too, e.g. $K_{0} z / h \cdot(1-z / H)^{2}$, and similar results are obtained (not shown).

\footnotetext{
${ }^{*}$ But the equality does not have to hold, as it does not in Oerlemans (1998).
} 

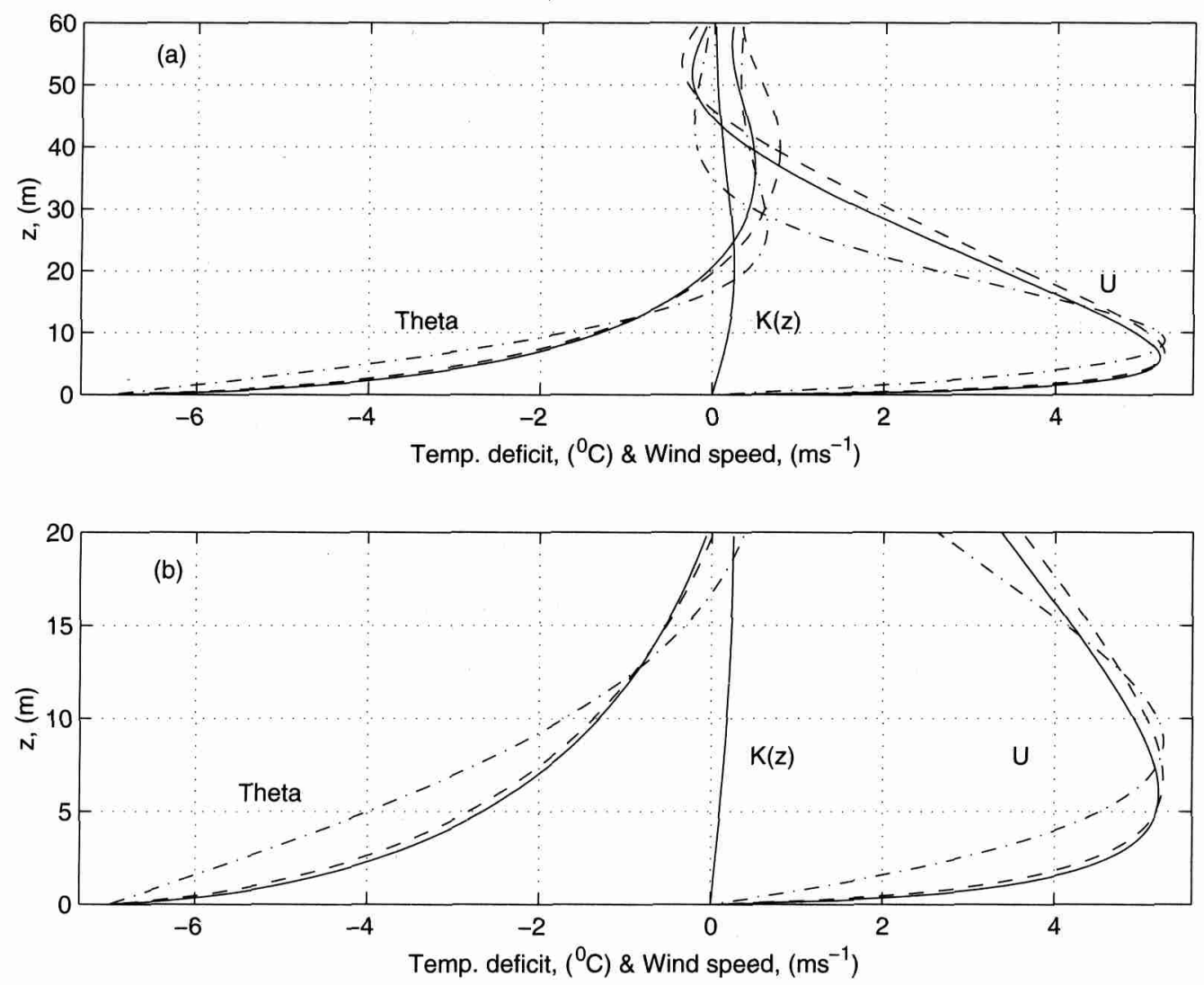

Figure 2. Comparison between the numerical (full lines), constant- $K$ (dot-dashed lines) and WKB (dashed lines) solutions. While (a) shows the whole stable boundary layer, (b) enlarges its lower part. Mostly left is $\Theta(z)$ while mostly right is $u(z)$. The eddy diffusivity/conductivity $K(z)$ from (3.1) has its maximum $0.25 \mathrm{~m}^{2} \mathrm{~s}^{-1}$ at $h=20 \mathrm{~m}$ (full lines). Other parameters: $\alpha=5^{\circ}, \gamma=6 \mathrm{~K} \mathrm{~km}^{-1}, C=-7 \mathrm{~K}, P r=1.1$, and constant- $K$ solution uses 0.3 $\max [K(z)]$. See text for explanation of symbols.

\section{(b) Data comparison}

Detailed eight-level mast measurements were obtained, besides eddy flux and balloon observations, over a valley glacier in the Alps during PASTEX (Pasterze experiment) in the summer of 1994 (e.g. Greuell et al. 1997). This is a good location for persistent katabatic flows. Here the observations from PASTEX, Austria 1994 (Van den Broeke 1997; Oerlemans 1998) are compared against the WKB and constant- $K$ solutions. Figure 3 displays an example of a katabatic flow. Note that the katabatic jet, i.e. the wind speed maximum, is imbedded into the surface inversion (important for fluxes) even though the strongest part of the inversion is actually below $z_{\mathrm{J}}$. Only the WKB solution compares favourably with the observed data. This is thoroughly discussed by GO where slightly different input parameters are assigned (see their Fig. 3). In GO some balloon data are used but not here, as they do not add anything to this surface flux estimation. The reliability of the GO model is supported by Denby (1999) who uses a 'heavy-duty' second-order turbulence closure numerical model for the same reason as here, and finds no better agreement with the data than shown in Fig. 3. In the following, the GO model reveals some dynamic details about the low-level jet, $K(z)$ and $C$, and relates these to the surface fluxes. 


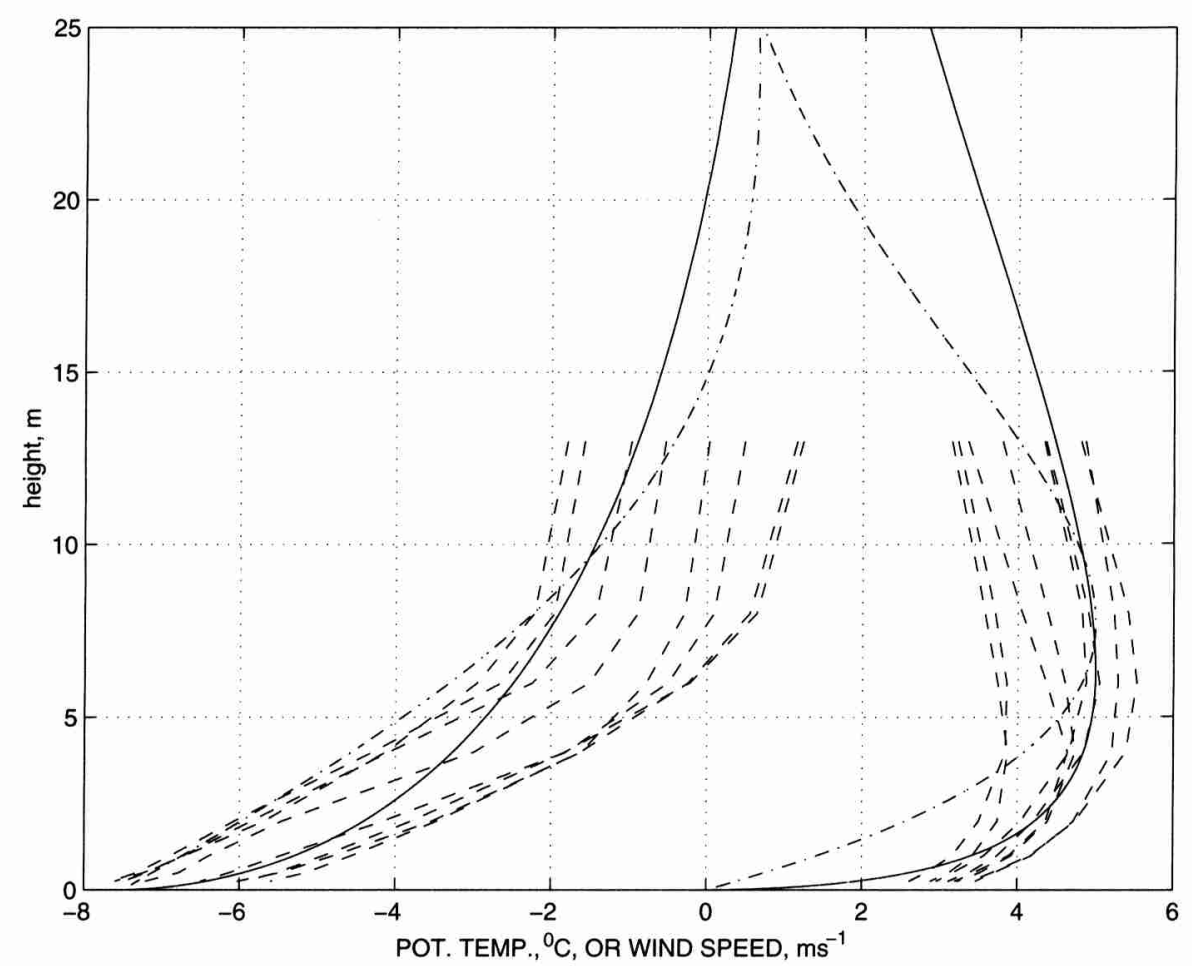

Figure 3. Comparison of $\Theta$ (potential-temperature deficit) and $u$ (katabatic wind) between: the WKB solution (full lines) and the observations on Pasterze (PASTEX, dashed lines), with the constant- $K$ solution (dot-dashed lines). The observations are 30-minute mean values for every three hours over a 24-hour period on 27 July 1994. The units are degC or $\mathrm{m} \mathrm{s}^{-1}$ (left and right, respectively). The generalized O'Brien $K(z)$ profile is used from (3.1) with $\max [K(z)]=0.30 \mathrm{~m}^{2} \mathrm{~s}^{-1}$ at $h=20 \mathrm{~m}$; other parameters: $\gamma=6 \mathrm{~K} \mathrm{~km}^{-1}, \alpha=0.1 \mathrm{rad}, C=-7.5 \mathrm{~K}$, $\operatorname{Pr}=1.4$, and constant $-K$ solution uses $0.2 \max [K(z)]$. See text for explanation of symbols.

\section{KATABATIC JET AND SURFACE FLUXES}

\section{(a) Definition}

Once $F$ is found in (2.5), the fluxes can be calculated since they are proportional to the derivative of $F$. Namely,

$$
\begin{aligned}
\left(\Theta_{*} u_{*}, u_{*}^{2}\right) & \equiv\left(K_{\mathrm{H}}\left(\frac{\mathrm{d} \Theta}{\mathrm{d} z}+\gamma\right), K_{\mathrm{M}} \frac{\mathrm{d} u}{\mathrm{~d} z}\right)_{\mathrm{DIM}} \\
& =C K\left(\frac{\mathrm{d} F}{\mathrm{~d} z}\right)_{\text {TOT }}(1, \operatorname{Pr} \mu)^{\mathrm{T}}
\end{aligned}
$$

where $\Theta(z)$ and $u(z)$ are dimensional again, $F$ is written as a row vector in (4.1b) where the first element includes the background stratification too (e.g. Stull 1988) - that is why the subscript TOT-and the superscript T denotes 'transposed'. The right-hand side (RHS) of (4.1) is evaluated near the surface. Note that if (4.1) is taken exactly at $z=0$, then $K=0$ too, and the gradient $(\mathrm{d} F / \mathrm{d} z)_{z=0}$ must tend to infinity, which is unrealistic, to give non-zero fluxes on the left-hand side of (4.1).

The only remaining point is how to connect the fluxes with the surface. The roughness length concept is invoked for. Consequently, the lowest meteorological level of interest becomes the assigned roughness length $z_{0}$ at which $K\left(z_{0}\right)$ is estimated from (3.1). For instance, if $z_{0} \sim 10^{-3} \mathrm{~m}$, then typically $K\left(z_{0}\right) \sim 10^{-4} \mathrm{~m}^{2} \mathrm{~s}^{-1}$. In this way the 
flux profiles become finite and nonzero at this new surface, $z_{0}$. It must be realized that in general $K\left(z_{0}\right)$ is a crude guess as well as $\left(\Theta_{*}, u_{*}\right)$ if directly related to the $z_{0}$ surface. To avoid such a gross flux presumption based only on (3.1) and (4.1), all evaluated at $z_{0}$, we use another approach-flux profile linear extrapolation downward from the katabatic low-level jet at $z_{\mathrm{J}}$ to $z_{0}$. Therefore, one expects these fluxes to be mainly dependent on $z_{\mathrm{J}}$ and only weakly on $z_{0}$. This is the main idea of this section.

\section{(b) The katabatic low-level jet}

Having obtained a sufficient agreement between the observed and the WKBmodelled katabatic flow, e.g. as in Fig. 3, the derivatives of $u$ and $\Theta$ can be obtained confidently. These will be used to extrapolate the turbulent fluxes $\left(\left\langle w^{\prime} \Theta^{\prime}\right\rangle,\left\langle u^{\prime} w^{\prime}\right\rangle\right)$, where $\langle\cdots\rangle$ indicates ensemble average, from $z_{\mathrm{J}}$ to $z_{0}\left(u_{*}^{2} \equiv-\left\langle u^{\prime} w^{\prime}\right\rangle\right.$ at $\left.z_{0}\right)$. Because at $z_{\mathrm{J}}$ the wind has its maximum, $\mathrm{d} u / \mathrm{d} z=0$ there; hence, $\left\langle u^{\prime} w^{\prime}\right\rangle=0$ there too, but not the local linear trend of $\left\langle u^{\prime} w^{\prime}\right\rangle$ which is nonzero.

Before proceeding with this now very simple procedure to obtain $\left(\Theta_{*} u_{*}, u_{*}^{2}\right)$, a few interesting details about the modelled flow are shown. From (2.5) evaluated at $z_{\mathrm{J}}$ where $\mathrm{d} u / \mathrm{d} z=0$ one finds:

$$
\tan \left\{\left(\frac{\sigma_{0}}{2}\right)^{1 / 2} \operatorname{Int}\left(z_{\mathrm{J}}\right)\right\}=1
$$

where Int denotes the integral in the exponent of (2.5),

$$
\operatorname{Int}(z) \equiv \int_{z_{0}}^{z} K^{-1 / 2} \mathrm{~d} z
$$

now evaluated between $z_{0}$ and $z_{\mathrm{J}}$ and neglecting its exceedingly small value at the former; hence,

$$
\operatorname{Int}\left(z_{\mathrm{J}}\right)=\left(\frac{2}{\sigma_{0}}\right)^{1 / 2} \frac{\pi}{4} .
$$

This integral property (4.2) is linked to $P r, \gamma$ and $\alpha$. Using (2.2b) for $\sigma_{0},\left\{\operatorname{Int}\left(z_{\mathrm{J}}\right)\right\}^{2}$ becomes inversely proportional to $N_{\alpha}$, i.e.

$$
\left\{\operatorname{Int}\left(z_{\mathrm{J}}\right)\right\}^{2}=\frac{\pi^{2}}{8} \frac{(\operatorname{Pr})^{1 / 2}}{N_{\alpha}},
$$

and any $K(z)$ contained in $\operatorname{Int}\left(z_{\mathrm{J}}\right)$ must comply to the physical constraint on the RHS of (4.2d): there is a balance between $z \mathrm{~J}, N_{\alpha}$ and a cumulative $K$. An increase in $N_{\alpha}$ (either through $\gamma$ or $\alpha$ ) causes a decrease in $\operatorname{Int}\left(z_{\mathrm{J}}\right)$ through either lowering $z_{\mathrm{J}}$ or/and suitably modifying, generally increasing, a prescribed $K(z)$. The particular form of the inverse of $K$ accumulated up to $z_{\mathrm{J}}$ is proportional to the 'slope projection' of the background buoyancy period, $2 \pi / N_{\alpha}$; in other words, the value of this integral form is decided by the assigned stability and terrain. The presence of a katabatic jet for arbitrarily large 'projected' buoyancy periods implies generally very small $K$-values. Also note that various $K(z)$ profiles may satisfy the same $z_{\mathrm{J}}, N_{\alpha}$ and $P r$. It is a cumulative $K$-value that matters in the balance described in (4.2).

Let us see some consequences of very simple choices for $K(z)$; this is important for modelling purposes (e.g. Mahrt 1998). Examine the oversimplified katabatic models for fluxes with (i) $K=K_{\mathrm{C}}=$ constant, or (ii) $K(z)=k u_{*} z$. One finds respectively: (i) the 
known relation between $z_{\mathrm{J}}$ being a large part of the classical katabatic SBL depth (e.g. Oerlemans 1998),

$$
z_{\mathrm{J}}=\frac{\pi}{4}\left(\frac{2 K_{\mathrm{C}}(P r)^{1 / 2}}{N_{\alpha}}\right)^{1 / 2}=\frac{\pi}{4} H_{\text {CLASSIC }}
$$

where $H_{\text {CLASSIC }}$ is the SBL classical depth $\left(\approx 1 / \sigma_{\mathrm{C}}\right)$, or (ii)

$$
u_{*}=\frac{32 N_{\alpha} z_{\mathrm{J}}}{k(\operatorname{Pr})^{1 / 2} \pi^{2}} .
$$

Both expressions (4.3), though qualitatively revealing, are inadequate for surface flux analyses because (i) $z_{\mathrm{J}}$ is wrongly placed around the mid-upper part of the SBL, or (ii) $u_{*}$ estimated linearly from true $z_{\mathrm{J}}(\sim 5 \mathrm{~m}$ or so) is typically too small. Of course, other approaches than in this study are possible* as in Denby and Smeets (2000). Because (4.3b) is physically based, it suggests a way to parametrize the minimum friction velocity, $\min \left(u_{*}\right)$, in large-scale numerical models to prevent a total surfacelayer decoupling (instead of an ad hoc code statement for $\min \left(u_{*}\right)$ ). Note that (4.2) is a generalization of the special case (4.3a).

Now $\operatorname{Int}\left(z_{\mathrm{J}}\right)$ is calculated for the chosen $K$. Using (4.2b) and (3.1), one obtains, integrating by parts,

$$
\operatorname{Int}\left(z_{\mathrm{J}}\right)=2\left(\frac{h z}{\mathrm{e}^{1 / 2} \max (K)}\right)^{1 / 2} \exp \left(\frac{(z / h)^{2}}{4}\right) \sum_{n=0}^{\infty}(-1)^{n}\left(\frac{z}{h}\right)^{2 n}\left(\frac{1}{4 n+1}\right) ! !
$$

where the RHS is evaluated for $z$ at $z_{\mathrm{J}}$ minus that at $z_{0}$ (the latter may be dropped again). Since the series in (4.4) converges rapidly, a reasonable estimate may result using only a single term! Simplifying it even more by replacing the exponential factor with one (i.e. $\left.z_{\mathrm{J}} \ll h<H\right),(4.2 \mathrm{~d})$ and (4.4) yield a useful approximate relation between $\max [K(z)]$, $h, z_{\mathrm{J}}$, and $\sigma_{0}\left(N_{\alpha}, \operatorname{Pr}\right)$ from (2.2b), namely,

$$
\max [K(z)] \approx 32 N_{\alpha} h z_{\mathrm{J}} \pi^{-2}(\mathrm{e} P r)^{-1 / 2} .
$$

The importance of (4.2), and especially the consequence (4.5) due to (3.1), is providing an estimate for $\max [K(z)]$ from an observed/expected height of the katabatic jet, guessed $h$ based on the observed/expected wind and temperature profiles, coupled with $N_{\alpha}$ and the WKB-requirement for $h$.

\section{(c) Downward extrapolation of the fluxes}

While $\left\langle u^{\prime} w^{\prime}\right\rangle$ will be extrapolated linearly from $z_{\mathrm{J}},\left\langle w^{\prime} \Theta^{\prime}\right\rangle$ will be taken as constant from $z_{\mathrm{J}}$ downward. The reasoning is the following. Intuitively speaking, this is in accord with the presence of the low-level katabatic jet centred at $z_{\mathbf{J}}$ (governed by both the wind speed gradient and curvature, i.e. $\mathrm{d} u / \mathrm{d} z$ and $\mathrm{d}^{2} u / \mathrm{d} z^{2}$ ) and imbedded into a surface inversion (determined by the nearly constant $\mathrm{d} \Theta / \mathrm{d} z$ ). The qualitative behaviour of the fluxes in the large part of the lower SBL, $z_{0} \ll z<h$, can be estimated from the expansions of $(2.3 \mathrm{a}, \mathrm{b})$, or more precisely from (2.5). The former suggests that $\left\langle u^{\prime} w^{\prime}\right\rangle$ behaves as the normalized $\mathrm{d}\left(u / u_{\max }\right) / \mathrm{d} \zeta \sim 1-2 \zeta$ and $\left\langle w^{\prime} \Theta^{\prime}\right\rangle \sim \mathrm{d}(\Theta / C) \mathrm{d} \zeta \sim$ $1-\zeta^{2}$, where $\zeta \equiv z / h, 0<\zeta<1$. The normalized expansions for the fluxes suggest that $\left\langle u^{\prime} w^{\prime}\right\rangle$ varies more than $\left\langle w^{\prime} \Theta^{\prime}\right\rangle$ in the lower SBL. Hence, if $\left\langle u^{\prime} w^{\prime}\right\rangle$ is treated as 
a linear function, it is not unreasonable to try $\left\langle w^{\prime} \Theta^{\prime}\right\rangle$ as a constant in the lower SBL instead of approximating its weak and nonlinear variability.

Van der Avoird and Duynkerke (1999) use both profile and sonic measurements to retrieve the fluxes in a katabatic flow. They conclude that the presence of the lowlevel katabatic jet affects only $\left\langle u^{\prime} w^{\prime}\right\rangle$ but not $\left\langle w^{\prime} \Theta^{\prime}\right\rangle$, and that $z_{\mathrm{J}}$ must enter the scaling for $\left\langle u^{\prime} w^{\prime}\right\rangle$; their points are not in conflict with our flux extrapolation. Smeets et al. (2000) address the fluxes from PASTEX. They indicate that $\left\langle u^{\prime} w^{\prime}\right\rangle$ can be extrapolated linearly down from $z_{\mathrm{J}}$ but $\left\langle w^{\prime} \Theta^{\prime}\right\rangle$ is more complicated. The latter seems to be a nearsurface constant, $\left\langle w^{\prime} \Theta^{\prime}\right\rangle_{0}$, for roughly $z<z_{\mathrm{J}} / 2$ decreasing linearly to its $50 \%$ at $z_{\mathrm{J}}$ and then remaining constant aloft -all within significant data scatter, see their Fig. 15 for normalized fluxes (no actual values shown). This suggests that our $\left(\Theta_{*} u_{*}\right)$ estimation would be 50\% too low. However, Smeets et al. (2000) in the same Fig. 15(b) also display the numerical result of the Denby (1999) prediction for $\left\langle w^{\prime} \Theta^{\prime}\right\rangle$ which at $z_{\mathrm{J}}$ remains around $80-85 \%$ of its $\left\langle w^{\prime} \Theta^{\prime}\right\rangle_{0}$. In other words, the second-order closure model of Denby (1999) gives nearly a constant value of $\left\langle w^{\prime} \Theta^{\prime}\right\rangle$ below $z_{\mathrm{J}}$, it is in accord with the reasoning in the former paragraph and it is in favour of our simple extrapolation. In short, there is an overall agreement that the katabatic $\left\langle u^{\prime} w^{\prime}\right\rangle$ varies almost linearly while its $\left\langle w^{\prime} \Theta^{\prime}\right\rangle$ counterpart varies significantly less with height below $z_{\mathrm{J}}$.

The momentum flux is approximated linearly from $z_{\mathrm{J}}$ to the surface, i.e.

$$
u_{*}^{2}=\frac{\mathrm{d}\left(-\left\langle u^{\prime} w^{\prime}\right\rangle\right)}{\mathrm{d} z} z_{\mathrm{J}}, \quad \mathrm{d} / \mathrm{d} z \text { taken at } z_{\mathrm{J}} .
$$

The final expression for $u_{*}$ using (4.1), (4.2a) and (4.6) is

$$
u_{*}=\left\{|C|(\operatorname{Pr} / 2)^{1 / 2} \mu N_{\alpha} z_{\mathrm{J}}\right\}^{1 / 2} \mathrm{e}^{-\pi / 8} .
$$

This estimation implicitly neglects a thin classic surface layer where $u_{*}^{2}$ should be height independent. The existence of such a layer that would have its thickness of the order of $1 \mathrm{~m}$ is questionable (e.g. Oerlemans 1998; Denby 1999), and here it is good that we avoid any direct influence from it and the vicinity of $K\left(z_{0}\right)$. Here, $z_{0}$ is used only tentatively to close the flux profiles at the surface; the fluxes are actually estimated based on $z_{\mathrm{J}}$.

The surface heat flux or, more precisely, just the product $\left(\Theta_{*} u_{*}\right)$ from (4.1) is treated, once again, as a constant $-\left\langle w^{\prime} \Theta^{\prime}\right\rangle$ downward from $z_{\mathrm{J}}$. Finally,

$$
\Theta_{*}=\left[\gamma K\left(z_{\mathrm{J}}\right)+|C|\left\{N_{\alpha} \operatorname{Pr}^{-1 / 2} K\left(z_{\mathrm{J}}\right)\right\}^{1 / 2} \mathrm{e}^{-\pi / 4}\right] / u_{*}
$$

where the second term dominates strongly; hence, using (4.7) and dropping the first term on the RHS of (4.8a):

$$
\Theta_{*} \approx\left(\frac{2^{1 / 2} K\left(z_{\mathrm{J}}\right)|C|}{\operatorname{Pr} \mu z_{\mathrm{J}}}\right)^{1 / 2} \mathrm{e}^{-\pi / 8} .
$$

This (4.8b) is expected to underestimate the total $\Theta_{*}$ in (4.8a) by up to a few per cent. That is how the surface flux parameters are determined-the goal of this section and the paper.

To summarize, since the WKB solution offered the derivatives to be readily found, obtaining the fluxes was a question of a further calculating exercise and the appropriate downward extrapolation. The latter is governed by the interplay between the katabatic jet and the surface inversion. Because the jet is imbedded in this inversion here, which is a common but not the definitive SBL case, the jet appears as the most important dynamical feature for the surface fluxes. This role of the katabatic jet for the SBL in terms of the TKE and stability functions is discussed by Van der Avoird and Duynkerke (1999), Denby (1999) and Smeets et al. (2000). 


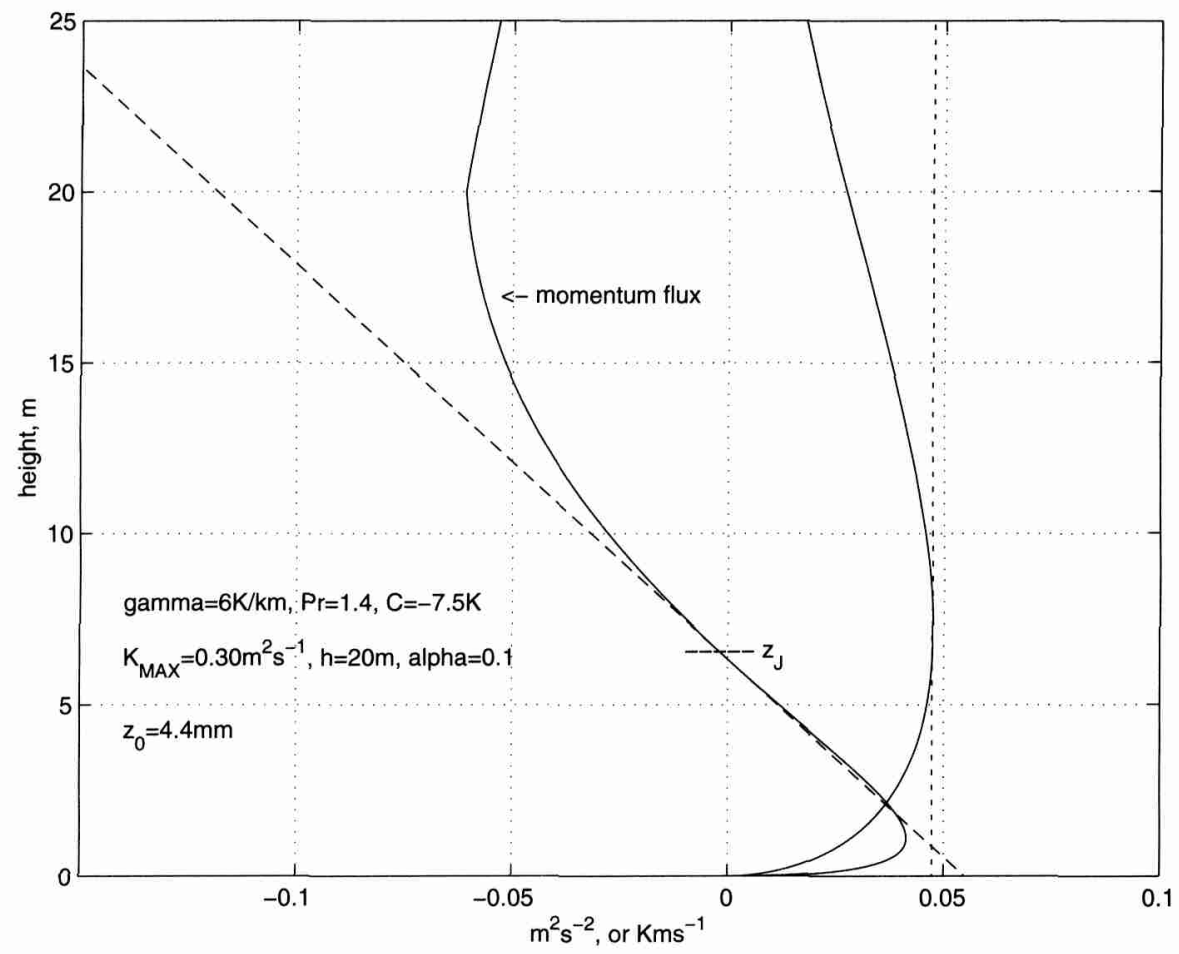

Figure 4. Surface flux estimation based on the modelled data from Fig. 3 and adopting roughness length $z_{0}=4.4 \mathrm{~mm}$. The calculated low-level jet height, $z_{\mathrm{J}}$, is at $6.34 \mathrm{~m}$. Full lines represent the momentum and temperature fluxes while the dashed and short-dashed (vertical) lines depict the way these fluxes are extrapolated from the jet down to the surface respectively $\left(0.0549 \mathrm{~m}^{2} \mathrm{~s}^{-2}, 0.0473 \mathrm{~m} \mathrm{~s}^{-1} \mathrm{~K}\right)$. The related surface parameters, $u_{*}$ and $\Theta_{*}$ compare well with those obtained experimentally or simulated by other authors within several per cent of the relative difference (see text).

\section{(d) An example}

Figure 4 shows the WKB flux profiles based on (4.1) that correspond to Fig. 3. These fluxes explain and justify the approach considered in this section. Denby (1999) compares his simulations of the mean profiles and the fluxes with the data from PASTEX in his Fig. 3. The agreement is within the measurement error bars or closer. From Denby's Fig. 3 one retrieves his $\left.\left\langle u^{\prime} w^{\prime}\right\rangle\right|_{0} \approx-0.048 \mathrm{~m}^{2} \mathrm{~s}^{-2}$ and $\left.\left\langle w^{\prime} \Theta^{\prime}\right\rangle\right|_{0} \approx-0.046 \mathrm{~K} \mathrm{~m} \mathrm{~s}^{-1}$, yielding $\left(\Theta_{*}, u_{*}\right)=\left(0.21 \mathrm{~K}, 0.22 \mathrm{~m} \mathrm{~s}^{-1}\right)$. With the method described in this section we obtain $\left(\Theta_{*}, u_{*}\right)=\left(0.202 \mathrm{~K}, 0.234 \mathrm{~m} \mathrm{~s}^{-1}\right)$; the relative differences are better than $-7 \%$. Our results also agree with Denby and Smeets (2000).

Following Munro (1989), Van den Broeke (1997) inferred the average $\Theta_{*}$ and $u_{*}$ for the data shown. Their iterative procedure (because fluxes depend on the mean gradients nonlinearly) fits the surface parameters to the observed profiles, and gives $\left(\Theta_{*}, u_{*}\right)=\left(0.21 \mathrm{~K}, 0.24 \mathrm{~m} \mathrm{~s}^{-1}\right)$. Although we criticize in the introduction such types of method, it gave a similar answer to Denby (1999) because this bulk method still works reasonably well over smooth glaciers such as Pasterze which is considered here (e.g. Smeets and Vugts 2001). Van den Broeke's (1997) calculation and our method both use $z_{0}=4.4 \mathrm{~mm}$; the relative differences between his and our $\left(\Theta_{*}, u_{*}\right)$ are less than $4 \%$. Denby (1999) uses $2 \mathrm{~mm}$ and $0.04 \mathrm{~mm}$ for the momentum and heat roughness length, respectively, (values closer to those in Smeets et al. (2000)). 
After analysing more data, one would come up with a more robust and maybe more general relation between $\left(\Theta_{*}, u_{*}\right)$ and the background variables-that is exactly what is needed for parametrizations of the SBL in numerical models. Thus, it is hoped the method proposed here will help to improve model parametrizations of katabatic flows.

\section{CONCLUSIONS}

The Prandtl model for katabatic flows with gradually varying $K(z)$ is used for the estimation of the main surface flux parameters, $\Theta_{*}$ and $u_{*}$. The model employing the WKB method is described here, and fully by Grisogono and Oerlemans (2001; throughout GO). A chosen $K(z)$ profile, besides its physical relevance and realizability, must satisfy the WKB condition, i.e. $K(z)$ varying gradually compared with the calculated quantities. It was shown by GO and here for the PASTEX data, Austria, 1994, that the new WKB solution resembles the data much better than the constant- $K$ solution. Meanwhile, the relative simplicity of the WKB solution, that is also checked against a numerical one, still gives some new insights into the dynamics of katabatic flow. For instance, an integral relationship between $K(z)$ and the katabatic jet level is found. Since the WKB method provides reliable vertical derivatives of the calculated perturbations, it is convenient to use these for the momentum and heat flux estimations. That is done here-the GO model is used to calculate $\Theta_{*}$ and $u_{*}$, and the results agree with the experimental and numerical flux estimations based on the data. The drawback of the analytical method used is a prescription of $K(z)$ which otherwise ought to be a function of the flow. That can be overcome in a numerical nonlinear model.

The heat flux varies much less with height than the momentum flux (see Figs. 3 and 4). For both flux profiles their very lowest parts (roughly $z \leq 1 \mathrm{~m}$ ) are discarded and extrapolations from the katabatic low-level jet height, $z_{\mathrm{J}}$, are used instead. The momentum flux is extrapolated linearly while the heat flux is simply taken as constant from $z_{\mathrm{J}}$ down to the surface since $z_{\mathrm{J}}$ is imbedded into the surface inversion; this is in a broad agreement with Van der Avoird and Duynkerke (1999) and our qualitative reasoning for $\mathrm{d} u / \mathrm{d} z$ and $\mathrm{d} \Theta / \mathrm{d} z$. The fluxes depend weakly on the particular value of $z_{0}$. Equations (4.7) and (4.8) give $u_{*}$ and $\Theta_{*}$. The relative differences between these main surface flux parameters obtained with the proposed method and those inferred from an iterative technique based on PASTEX observations (Van den Broeke 1997) and their benchmark numerical simulations (Denby 1999) are within several per cent. While the overall shape of the heat flux profile for katabatic flows is still debated and needs more measurements (Van der Avoird and Duynkerke 1999; Denby 1999; Smeets et al. 2000; Smeets and Vugts 2001), our simple surface heat flux evaluation agrees with those PASTEX values that are readily found in the literature. Beyond this question, the employed WKB method is able, at least in principle, to calculate the katabatic flux profiles for guessed $K(z)$ profiles.

More SBL data need to be processed and compared with the proposed method for the estimation of the surface fluxes. Hopefully, this study would yield a more thorough data interpretation, surface flux estimations and a better parametrization of the katabatic flows in large-scale numerical models which is not the case today. For modelling purposes, $\left(\Theta_{*}, u_{*}\right)$ needs to be coupled with a surface parametrization (surface cooling rate, $z_{0}, \alpha$, etc.) and dynamical subroutines providing $\gamma, \operatorname{Pr}$, background wind, etc.

\section{ACKNOWLEDGEMENTS}

Our colleagues at Stockholm, Uppsala and Utrecht universities are thanked for discussions. Constructive criticism from Michael Tjernström is appreciated. 
Arritt, R. W. and Pielke, R. A

1986

Bender, C. M. and Orszag, S. A.

Defant, F.

Denby, B.

Denby, B. and Smeets, C. J. P. P.

Durran, D. R.

Egger, J.

Gill, A. E.

Greuell, W., Knap, W. and Smeets, P.

Grisogono, B.

Grisogono, B. and Oerlemans, J.

Mahrt, L.

Munro, D. S.

Nappo, C. J. and Rao, K. S.

O'Brien, J. J.

Oerlemans, J.

1990

1982

1997

1995

2001

1982

1998

1989

1987

1970

1998

2000

Oerlemans, J. and Vugts, H. F.

1993

Oerlemans, J., Björnsson, H., Kuhn, M., Obleitner, F., Palsson, F., Smeets, P., Vugts, H. F. and de Wolde, J.

Pahlow, M., Parlange, M. B. and Porté-Agel, F.

Pielke, R. A.

Prandtl, L.

Smeets, C. J. P. P. and Vugts, H. F.

Smeets, C. J. P. P., Duynkerke, P. G. 1999 and Vugts, H. F.

Stull, R. B.

Van den Broeke, M. R.

\section{REFERENCES}

Interactions of nocturnal slope flows with ambient winds. Boundary-Layer Meteorol., 37, 183-195

Advanced mathematical methods for scientists and engineers. McGraw-Hill, Inc.

Zur theorie der Hangwinde, nebst bemerkungen zur Theorie der Bergund Talwinde. Arch. Meteorol. Geophys. Biokl. Ser., A1, $421-450$

Second-order modelling of turbulence in katabatic flows. Boundary-Layer Meteorol., 92, 67-100

Derivation of turbulent flux profiles and roughness lengths from katabatic flow dynamics. J. Appl. Meteorol., 39, 1601-1612

Numerical methods for wave equations in geophysical fuid dynamics. Springer

Thermally forced flows: theory. Pp. 43-57 in Atmospheric processes over complex terrain. Ed. W. Blumen. American Meteorological Society, Boston, USA

Atmosphere-ocean dynamics. Academic Press.

Elevational changes in meteorological variables along a midlatitude glacier during summer. J. Geophys. Res., 102(D22), 25941-25954

A generalized Ekman layer profile within gradually varying eddy diffusivities. Q. J. R. Meteorol. Soc., 121, 445-453

Katabatic flow: analytic solution for gradually varying eddy diffusivities. J. Atmos. Sci., 58, 3349-3354

Momentum balance of gravity flows. J. Atmos. Sci., 39, 27012711

Stratified atmospheric boundary layers and breakdown of models. Theoret. Comput. Fluid Dyn., 11, 263-279

Surface roughness and bulk heat transfer on a glacier. J. Glaciol., 35, 343-348

A model study of pure katabatic flow. Tellus. 39A, 61-71

A note on the vertical structure of the eddy exchange coefficient in the planetary boundary layer. J. Atmos. Sci., 27, 1213-1215

'The atmospheric boundary layer over melting glaciers'. Pp. 129153 in Clear and cloudy boundary layers. Royal Netherlands Academy of Arts and Sciences, Amsterdam.

Analysis of a three-year meteorological record from the ablation zone of the Morteratschgletscher, Switzerland: energy and mass balance. J. Glaciol., 46, 571-579

A meteorological experiment in the melting zone of the Greenland ice sheet. Bull. Am. Meteorol. Soc., 74, 355-365

1999 A glacio-meteorological experiment on Vatnajökull, Iceland, summer 1996. Boundary-Layer Meteorol., 92, 3-26

On Monin-Obukhov similarity in the stable atmospheric boundary layer. Boundary-Layer Meteorol., 99, 225-248

Mesoscale numerical modeling. Academic Press.

Führer durch die Strömungslehre. Pp. 373-375 in Vieweg und Sohn, Braunschwieg.

The evaluation of the surface energy balance and flux-profile relations over an Alpine and sea based glacier. J. Glaciol., in press

Observed wind profiles and turbulence fluxes over an ice surface with changing surface roughness. Boundary-Layer Meteorol., 92, 101-123

Turbulence characteristics of the stable boundary layer over a mid-latitude glacier. Part II: Pure katabatic forcing conditions. Boundary-Layer Meteorol., 97, 73-107

An introduction to boundary layer meteorology. Kluwer Academic Publishers

1997 Momentum, heat and moisture budgets of the katabatic wind layer ever a mid-latitude glacier in summer. J. Appl. Meteorol., 36, 763-774 
Van der Avoird, E. and Duynkerke, P. G.

Zilitinkevich, S. and Calanca, P.
1999 Turbulence in a katabatic flow. Does it resemble turbulence in stable boundary layers over flat surfaces? Boundary-Layer Meteorol., 92, 39-66

2000 An extended similarity theory for the stably stratified atmospheric surface layer. Q. J. R. Meteorol. Soc., 126, 1913-1923 